\title{
Underdiagnosis of early cervical cancer with an invisible cervical transformation zone in an elderly patient: A case report
}

\author{
MASAKO ISHIKAWA $^{1}$, KENTARO NAKAYAMA ${ }^{1}$, HITOMI YAMASHITA $^{1}$, \\ TOMOKA ISHIBASHI ${ }^{1}$, SEIYA SATO ${ }^{1}$, SONOMI KUROSE $^{1}$, KIYOKA SAWADA $^{1}$, \\ HIROKI SASAMORI ${ }^{1}$, SULTANA RAZIA ${ }^{1}$, NORIYOSHI ISHIKAWA ${ }^{2}$ and SATORU KYO ${ }^{1}$ \\ Departments of ${ }^{1}$ Obstetrics and Gynecology and ${ }^{2}$ Organ Pathology, \\ Shimane University Faculty of Medicine, Izumo, Shimane 6938501, Japan
}

Received December 27, 2019; Accepted June 7, 2021

DOI: $10.3892 / \mathrm{mco} .2021 .2412$

\begin{abstract}
The treatment of cervical intraepithelial neoplasia (CIN) can result in under- or overtreatment. The current report describes a case of undertreatment of a cervical tumor. A 72-year-old woman was preoperatively diagnosed with CIN3. Following surgery, the final diagnosis of the excised specimen was keratinizing squamous cell carcinoma that measured $2.5 \mathrm{~cm}$ in size. The exocervical margin and deep margin were negative. The patient received adjuvant therapy with concurrent chemoradiotherapy and never had disease recurrence. In elderly patients, making an accurate preoperative diagnosis based on specimens from cervical biopsies with or without colposcopy is difficult. MRI may be an accurate preoperative indicator of early cervical tumor, although some studies have demonstrated that MRI has a limitation with respect to its diagnostic ability. Other studies have reported that it is necessary to perform conization prior to hysterectomy. Physicians must reconsider the determined preoperative diagnosis of an early cervical tumor and establish standard guidelines for deciding when to use surgical treatment in elderly patients.
\end{abstract}

\section{Introduction}

Cervical cancer is the second most common cancer globally exceeding 275,000 deaths and 530,200 new cases in 2010 (1).

Correspondence to: Dr Kentaro Nakayama, Department of Obstetrics and Gynecology, Shimane University Faculty of Medicine, Enyacho 89-1, Izumo, Shimane 6938501, Japan

E-mail:kn88@med.shimane-u.ac.jp

Abbreviations: ASC-US, atypical squamous cells of undetermined significance; CIN, cervical intraepitherial neoplasia; TZ, transformation zone; LSIL, low-grade squamous intraepithelial lesion; HSIL, high-grade squamous intraepithelial lesion; MIS, microinvasive surgery

Key words: atypical squamous cells of undetermined significance, cervical intraepithelial neoplasia, cervical cancer, underdiagnosis, magnetic resonance imaging, preoperative diagnosis, total laparoscopic hysterectomy
Approximately 13,000 new cases of cervical cancer were diagnosed in Japan, and an estimated 3,500 women were died from this disease (2). To our knowledge, high-grade squamous intra epithelial lesions (HSILs) are able to progress to become invasive cervical cancer, the detection of HSIL plays a crucial role in preventing cervical cancer (3). Cervical carcinoma in young women is considered a social problem and is difficult to diagnose in elderly women (4-9).

In the previous report, Müller et al (5) described the accuracy of diagnosis between histological assessment of biopsy findings and the final histological result after conization. In their reports, complete agreement in between 45 and $89.6 \%$ of cases. Agreement within one histological grade was present in between 74 and $89.9 \%$ of cases (5-15). Other reports referred to the accuracy of histological diagnosis from the specimen taken by biopsy or cervical conization. There were different conclusions, one concluded about the good correlation between the histological results on colposcopically directed biopsy (CDB) and cone specimens, the other concluded that it was questioned (4). Fan et al (6) described the overall agreement between the histological results on biopsy and the corresponding cone specimens was $74.1 \%$; underestimation occurred in $6.4 \%$ of cases and concluded that their data suggest that old age $(\geq 50)$, postmenopausal status, and transformation zone type $3 \mathrm{might}$ be positively associated with the under-diagnosis of CDB.

As discussed already, it had been difficult to diagnose early cervical disease, and treatments of cervical intraepithelial neoplasia (CIN) sometimes results in under- or overtreatment. However, accurately diagnosing patients with a small tumor in the cervix based on both biopsy results and imaging findings of modalities, such as magnetic resonance imaging (MRI), may be possible. Cervical conization is also a suitable option for diagnosing difficult cases. Herein, we describe a case of undertreatment of a cervical tumor and discuss difficulties associated with managing these small tumors.

\section{Case report}

A 72-year-old woman, gravida 4, para 3, was referred to our hospital because of treatment for CIN. She had abdominal 
pain and abnormal vaginal bleeding. In a previous clinic, she was determined to have abnormal cervical cytology with atypical squamous cells of undetermined significance (ASC-US); therefore, she underwent colposcopy-guided biopsy (Figs. 1A, B and 2).

She was diagnosed with severe dysplasia 2 weeks before presenting to our hospital. In the physical examination, performing speculum examination was difficult owing to atrophic vaginitis. Colposcopy findings shew dense acetowhite epithelium (W2) with an irregular, geographic border in the 4-7-o'clock direction. Coarse mosaic (M2) was also observed in the 6-o'clock direction. In current case, because of the patient's pain complaint, it was difficult to perform punch biopsy; thus, the sample volume was small. Cytological examination revealed three-fold enlarged nuclei and mild aggregation of chromatin, but no koilocytosis, indicating the absence of low-grade squamous intraepithelial lesions (LSIL). Histological examinations showed atypical cells in all layers; however, the basal membrane was preserved. Furthermore, no invasive region was observed. Ultrasonography (US) findings also showed a normal-shaped uterine cervix. There was no doubt regarding tumor formation and the normal shape of the uterine cervix. Findings also showed there was no region doubt regarding the normal shape of the uterine cervix (Fig. 3). Finally, the patient was diagnosed with CIN3.

It was decided to perform total laparoscopic hysterectomy and bilateral salpingo-oophorectomy for CIN3. When the operative procedures were completed, we assessed the cervical canal of the uterus and detected a mass in her cervix measuring approximately $2 \mathrm{~cm}$. Ultimately, the pathological diagnosis was keratinizing squamous cell carcinoma measuring $2.5 \mathrm{~cm}$ in size that was staged pT1b2pNxM0 according to the TNM classification. The clinical stages of the case were identified according to the definition of the International Federation of Gynecology and Obstetrics (FIGO) as stage IB2. The exocervical margin and deep margin were negative; however, lymphovascular invasion was noted (Fig. 4A and B). The time period from punch biopsy to laparoscopic surgery was 4 months.

On the grounds of these findings, we determined that she was preoperatively underdiagnosed. Therefore, it was recommended to undergo concurrent chemoradiotherapy (CCRT) that was necessary to prevent disease recurrence because the previous operation could not have been sufficient for stage IB2 cervical carcinoma. The patient and her family agreed with our recommendation, and she received CCRT (50 Gy of whole pelvic radiotherapy with 6 cycles of weekly $40 \mathrm{mg} / \mathrm{m}^{2}$ cisplatin) (Fig. 5). No other treatment was administered, and over a 3-year follow-up, she did not demonstrate any recurrence. We describe the details of the current case in Table I.

\section{Discussion}

We experienced a difficult case in which cytological findings indicated ASC-US, histological findings of the biopsy specimen indicated severe dysplasia, and histological findings of the postoperative specimen indicated invasive carcinoma. Finally, the patient was diagnosed with stage IB2 cervical carcinoma. We analyzed cases from our
Table I. Clinicopathological characteristics of the current case.

\begin{tabular}{ll}
\hline Characteristic & \multicolumn{1}{c}{ Current case } \\
\hline Age, years & 72 \\
Histology & Keratinizing squamous \\
& cell carcinoma \\
FIGO stage & Not applicable \\
UICC stage & pT1b1pNxpMx \\
Tumor size, cm & 2.5 \\
LVSI & Positive \\
Metastases paraaortic LN (CT) & Negative \\
Metastases pelvic LN (CT) & Negative \\
Metastases distance (CT) & Negative \\
Treatment & Operation+adjuvant \\
& (CCRT) \\
Recurrence within 3 years & None \\
Death within 3 years & None
\end{tabular}

FIGO, Federation of Gynecology and Obstetrics; UICC, Union for International Cancer Control; LVSI, lymphovascular invasion; LN, lymph node; CCRT, concurrent chemoradiotherapy; CT, computed tomography.

institute in which the cytological and histological results did not match. In the past 3 years, 123 of 4780 patients (2.6\%) have been diagnosed as having ASC-US, of whom 20 patients $(1.6 \%)$ were diagnosed accurately, 17 were diagnosed with LSIL/CIN1, and 3 were diagnosed with HSIL/CIN3. Among HSIL cases, one patient was diagnosed with ASC-US during pregnancy, followed by a diagnosis of HSIL during the puerperal period. Our elderly patient was diagnosed with CIN3 and had an invisible type 3 cervical transformation zone (TZ).

After we analyzed these cases, we found that ASC-US tended to be underdiagnosed. In elderly women, obtaining a sample of the diseased lesion may be difficult, possibly leading to underdiagnosis. Furthermore, it was already known that in women aged $>50$ years, the findings of colposcopy cannot be adequately assessed (16). If a TZ is not visible or only partially visible on colposcopy, the examination may miss areas where changes have occurred, and these changes will not be biopsied. If performed correctly, endocervical cytology should be able to capture existing cellular changes (17). Furthermore, regarding histological examinations by punch biopsy, Stuebs et al (18) reported that the rate of underdiagnosis was worse in women with type $3 \mathrm{TZ}$. Müller et al (5) also demonstrated that the agreement between biopsy findings and results after conization was significantly affected by patient age and that the agreement between preoperative findings and final histological results was only $49.1 \%$ for early cervical cancer. Women aged $>50$ years are more likely to be postmenopausal and have a type $3 \mathrm{TZ}$, which results in a higher rate of colposcopies, which are difficult to assess, and a lower rate of agreement between the diagnosis based on colposcopy and histological results $(19,20)$. However, sometimes, it could be diagnosed similarly between findings of cervical biopsy and those of cervical conization specimen (5), 


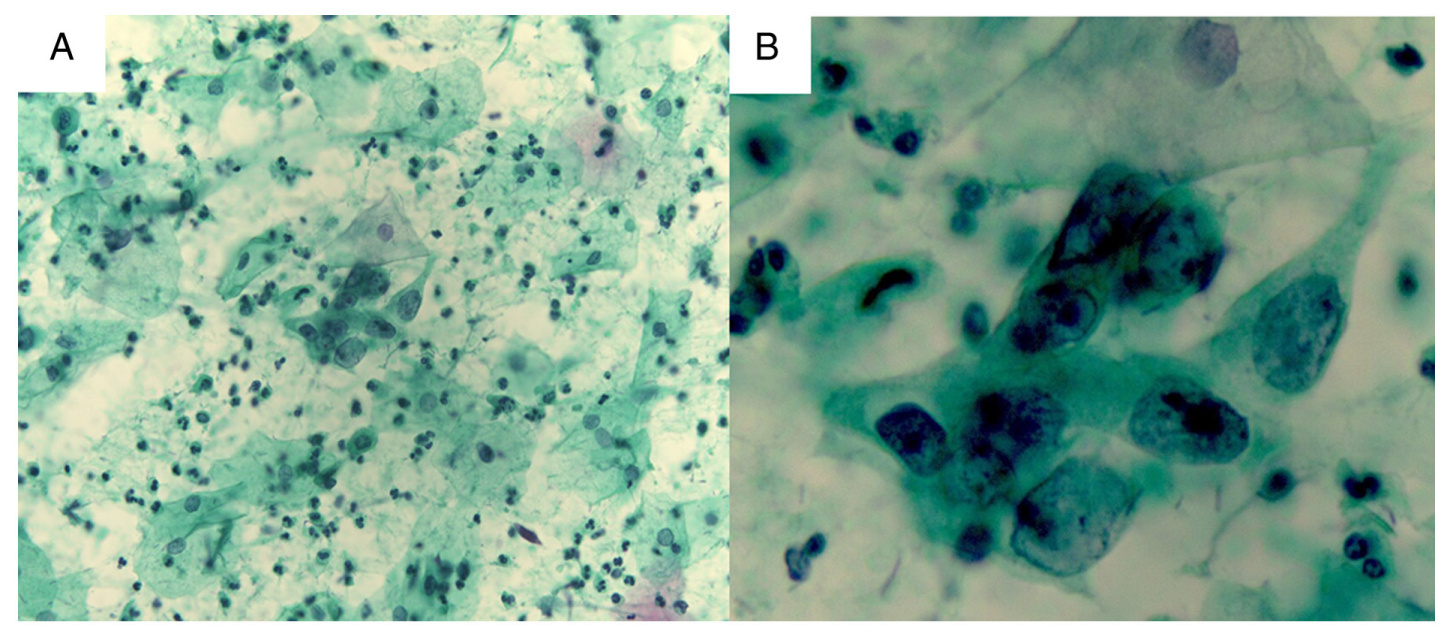

Figure 1. (A) Cytological findings of atypical squamous cells of undetermined significance; there are three-fold enlarged nuclei and mild aggregation of chromatin (low-power field; magnification, x40). (B) Cytological findings of ASC-US and cervical intraepithelial neoplasia 3. There is mild aggregation of chromatin but no koilocytosis, indicating the absence of low-grade squamous intraepithelial lesions. The patient's diagnosis is ASC-US (high-power field; magnification, x100). ASC-US, atypical squamous cells of undetermined significance.

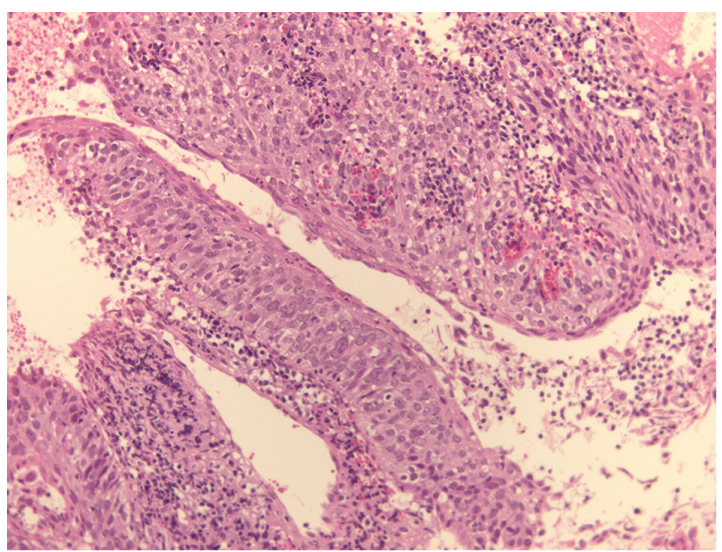

Figure 2. Histological findings of punch biopsy. Atypical cells are observed in all layers, but the baseline membrane is preserved. The patient's diagnosis is cervical intraepithelial neoplasia 3. No invasive regions are observed (low-power field; magnification, x40).

it is difficult for physicians to locate the diseased lesion on the deep in cervical canal in elderly women. Therefore, the biopsy results are not acceptable for making the final diagnosis. In elderly women, lesions of the squamocolumnar junction move up to the endocervical canal; therefore, it is sometimes very challenging to detect the diseased lesion of CIN and obtain the correct specimen for diagnostic purposes (6). According to current case, we established a procedure in which patients who were diagnosed with CIN3 based on punch biopsy results must undergo MRI. If the tumor mass comprises no diseased lesion, laparoscopic simple hysterectomy can be considered an operative procedure. MRI is a useful approach for detecting abnormal lesions in the cervix (21). Some studies reported that diffusion-weighed MRI can detect diseased lesions better than conventional MRI $(22,23)$, whereas other studies demonstrated that MRI has a limitation in its diagnostic ability (24). Hence, we reported that it is necessary to perform conization before hysterectomy $(5,6)$. We started preoperatively performing MRI for patients diagnosed with HSIL/CIN3, and

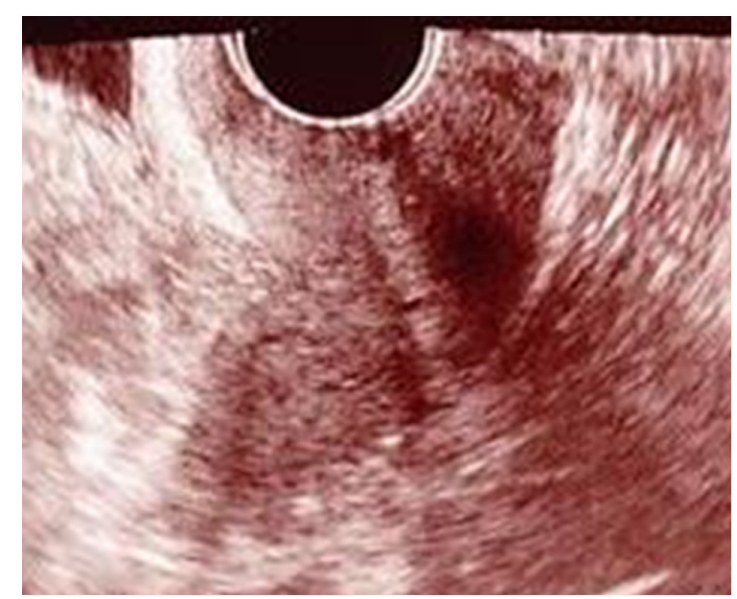

Figure 3. Ultrasound findings showing normal shape of the uterine cervix.

since this protocol has been established, no cases similar to the current case have been observed. The idea of performing conization before hysterectomy has been discussed; however, the gold standard guideline remains unknown. US may also help diagnose early cervical cancer, and although MRI is very useful for diagnosis, it is not commonly performed in developing countries. Therefore, contrast-enhanced computed tomography (CT) should be considered. In Japan, CT is commonly used for diagnosing early cervical cancer owing to the detection of lymph node metastasis. However, CT is not commonly used for CIN disease. In the current case, US was performed; however, the disease lesion was not detected. US may be a useful procedure for detecting disease lesions not only with B-mode image but also with color Doppler, although sometimes disease lesions cannot be clearly detected.

Microinvasive surgery (MIS) is common and most suitable procedure for early cervical lesions of carcinoma and CIN. The necessity for MIS has increased over the last several years; therefore, physicians must reconsider the determined preoperative diagnosis in cases similar to our case. Furthermore, in our country, although laparoscopic 
A

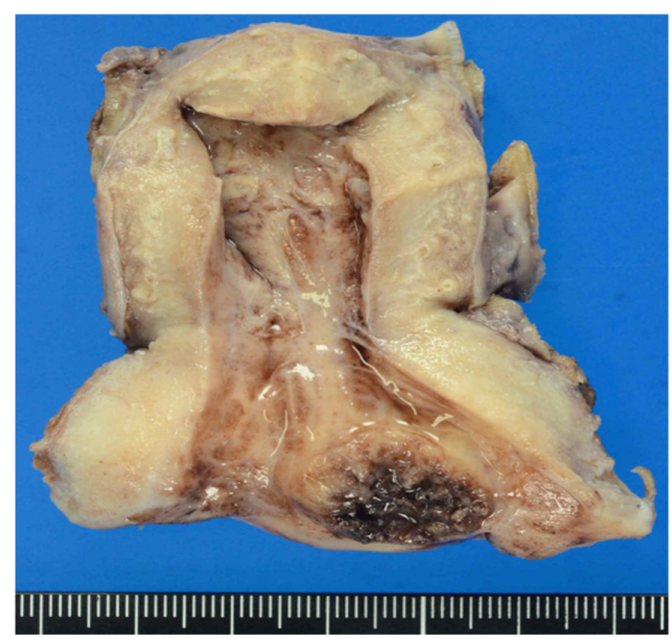

B

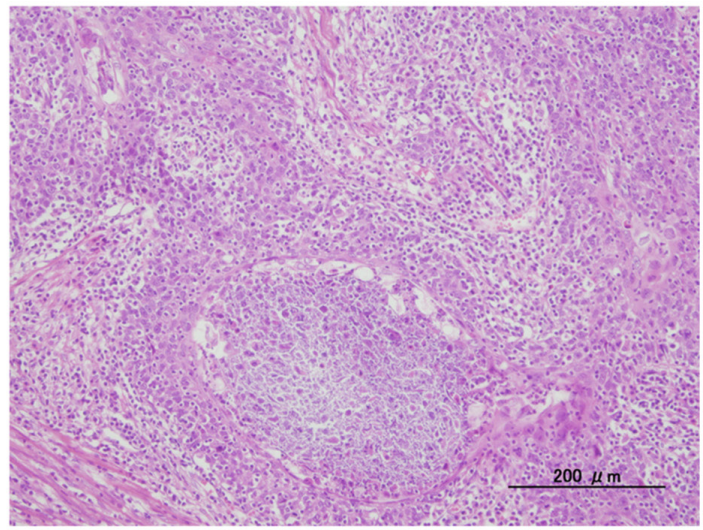

Figure 4. (A) Photograph of the resected portion of the uterus. A $2.5 \mathrm{~cm}$-sized tumor is located in the cervical canal and was determined to be keratinizing squamous cell carcinoma. The pathological diagnosis is pT1blpNxM0, stage 1B1 cervical carcinoma. (B) Isolated uterus for operation; Hematoxylin and eosin staining of the keratinizing squamous cell carcinoma. There were lymphovascular invasion (high-power field).

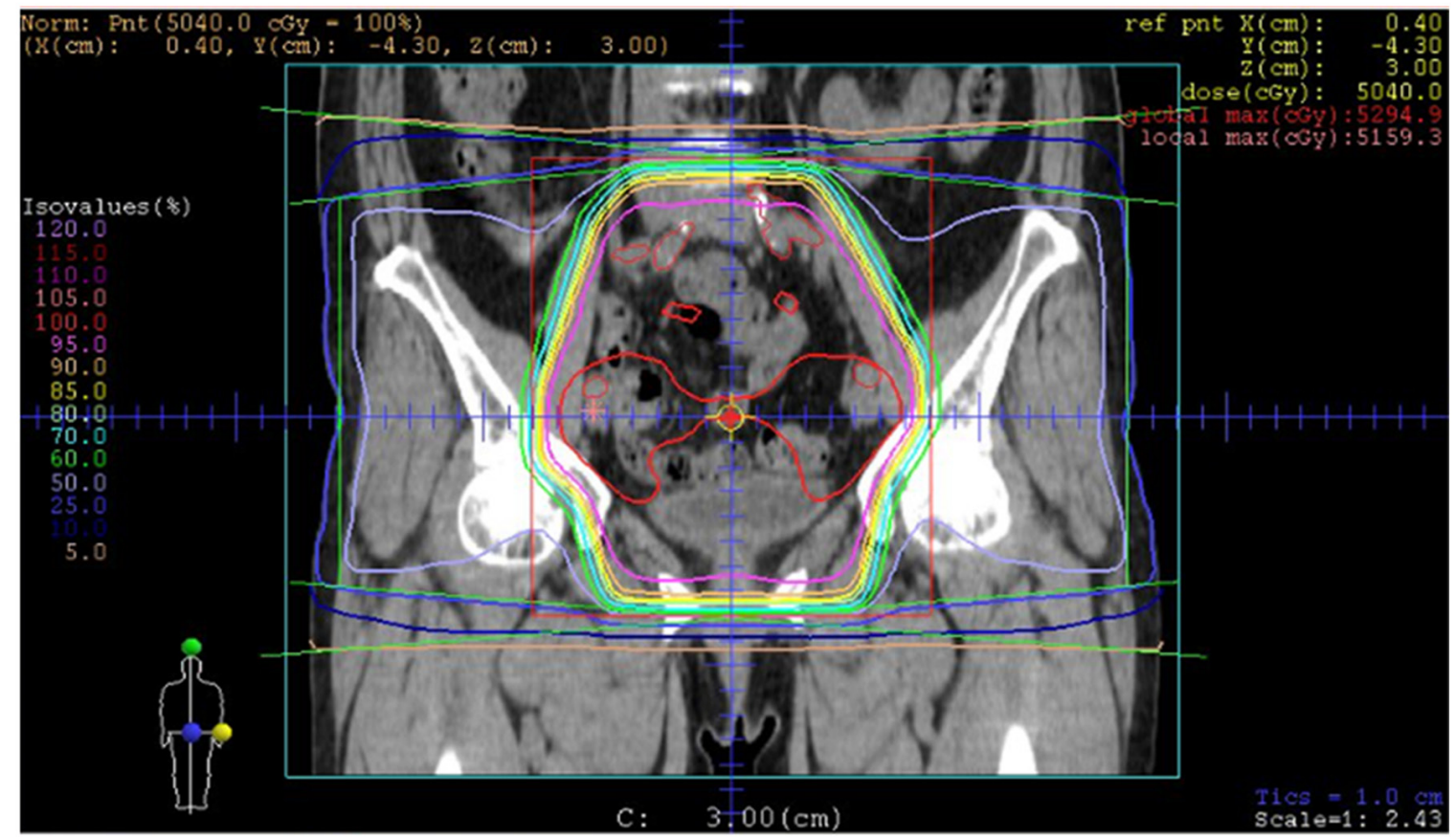

Figure 5. Radiotherapy field of the whole pelvis.

surgery is currently approved and covered by insurance for early cervical tumors such as HSIL/CIN3 and early cervical cancer, the recommended operative procedure is extremely different. Thus, it is imperative to improve preoperative diagnosis by utilizing useful tools so that the diagnosis is more accurate.

\section{Acknowledgements}

Not applicable.

\section{Funding}

No funding was received.

\section{Availability of data and materials}

The datasets used and analyzed during the current study are available from the corresponding author on reasonable request.

\section{Authors' contributions}

$\mathrm{KN}$ made the diagnosis and advised on manuscript preparation. MI made the diagnosis and drafted the manuscript. SR made substantial contributions to conception and design, and drafted the manuscript. HY contributed to analysis and interpretation of data. TI helped to collect the patient's data and corrected the manuscript. SS helped to collect the patient's data and was involved in critically revising the manuscript for important 
intellectual content. NI carried out the pathological diagnosis. $\mathrm{KS}$ and $\mathrm{SKu}$ treated the patient and helped to collect the patient's data. HS helped to collect the patient's data and helped with statistical analysis. SR and HY confirmed the authenticity of all the raw data. SKy made substantial contributions to the analysis and interpretation of data, advised on manuscript preparation and made revisions to the final manuscript. All authors have read and approved the final manuscript.

\section{Ethics approval and consent to participate}

The case report protocol was approved by the institutional ethics and research review boards at Shimane University (IRB nos. 20070305-1 and 20070305-2). In the application forms given to patients, for the patients who could not come to hospital, it was clearly stated that the opportunity to opt out was always available to patients. Information on opting out was stated on our website, or arrangements were made for patients to opt out.

\section{Patient consent for publication}

Patient consent for publication was obtained using an online opt-out approach.

\section{Competing interests}

The authors declare that they have no competing interests.

\section{References}

1. Ferlay J, Shin HR, Bray F, Forman D, Mathers C and Parkin DM: Estimates of worldwide burden of cancer in 2008: GLOBOCAN 2008. Int J Cancer 127: 2893-2917, 2010.

2. Hori M, Matsuda T, Shibata A, Katanoda K, Sobue T and Nishimoto H; Japan Cancer Surveillance Research Group: Cancer incidence and incidence rates in Japan in 2009: A study of 32 population-based cancer registries for the monitoring of cancer incidence in Japan (MCIJ) project. Jpn J Clin Oncol 45: 884-891, 2015.

3. Sasieni P and Adams J: Effect of screening on cervical cancer mortality in England and Wales: analysis of trends with an ageperiod cohort model. BMJ 318: 1244-1245, 1999.

4. Baldauf JJ, Dreyfus M, Ritter J and Philippe E: An analysis of the factors involved in the diagnostic accuracy of colposcopically directed biopsy. Acta Obstet Gynecol Scand 76: 468-473, 1997.

5. Müller K, Soergel P, Hillemanns P and Jentschke M: Accuracy of colposcopically guided diagnostic methods for the detection of cervical intraepithelial neoplasia. Geburtshilfe Frauenheilkd 76 : 182-187, 2016.

6. Fan A, Zhang L, Wang C, Wang Y, Han C and Xue F: Analysis of clinical factors correlated with the accuracy of colposcopically directed biopsy. Arch Gynecol Obstet 296: 965-972, 2017.

7. Chappatte OA, Byrne DL, Raju KS, Nayagam M and Kenney A Histological differences between colposcopic-directed biopsy and loop excision of the transformation zone (LETZ): A cause for concern. Gynecol Oncol 43: 46-50, 1991.

8. Zhang X, Dou Y, Wang M, Li Y, Wang F, Xie X and Wang X: A retrospective analysis on 1901 women with high grade cervical intraepithelial neoplasia by colposcopic biopsy. Eur J Obstet Gynecol Reprod Biol 217: 53-58, 2017.
9. Burger MP and Hollema H: The reliability of the histologic diagnosis in colposcopically directed biopsies. A plea for LETZ. Int J Gynecol Cancer 3: 385-390, 1993.

10. Barker B, Garcia F, Lozevski J, Warner J and Hatch K: The correlation between colposcopically directed cervical biopsy and loop electrosurgical excision procedure pathology and the effect of time on that agreement. Gynecol Oncol 82: 22-26, 2001.

11. Zuchna C, Hager M, Tringler B, Georgoulopoulos A, Ciresa-Koenig A, Volgger B, Widschwendter A and Staudach A: Diagnostic accuracy of guided cervical biopsies: A prospective multicenter study comparing the histopathology of simultaneous biopsy and cone specimen. Am J Obstet Gynecol 203: 321.e1-e6, 2010.

12. Kirkup W and Hill AS: The accuracy of colposcopically directed biopsy in patients with suspected intraepithelial neoplasia of the cervix. Br J Obstet Gynaecol 87: 1-4, 1980.

13. Heatley MK and Bury JP: The correlation between the grade of dyskaryosis on cervical smear, grade of cervical intraepithelial neoplasia (CIN) on punch biopsy and the final histological diagnosis on cone biopsies of the cervix. Cytopathology 9: 93-99, 1998.

14. Stubbe M, Broschewitz U, Kramm G, Schmidt W and Radtke A: Analysis of conizations performed 2005-2009 in Rostock. Geburtsh Frauenheilk 71: 187-193, 2011.

15. Duesing N, Schwarz J, Choschzick M, Jaenicke F, Gieseking F, Issa R, Mahner S and Woelber L: Assessment of cervical intraepithelial neoplasia (CIN) with colposcopic biopsy and efficacy of loop electrosurgical excision procedure (LEEP). Arch Gynecol Obstet 286: 1549-1554, 2012

16. UnderwoodM,ArbynM,Parry-Smith W,De Bellis-Ayres S, Todd R, Redman CW and Moss EL: Accuracy of colposcopy-directed punch biopsies: A systematic review and meta-analysis. BJOG 119: 1293-1301, 2012.

17. Benedet JL, Anderson GH and Boyes DA: Colposcopic accuracy in the diagnosis of microinvasive and occult invasive carcinoma of the cervix. Obstet Gynecol 65: 557-562, 1985

18. Stuebs FA, Schulmeyer CE, Mehlhorn G, Gass P, Kehl S, Renner SK, Renner SP, Geppert C, Adler W, Hartmann A, et al: Accuracy of colposcopy-directed biopsy in detecting early cervical neoplasia: A retrospective study. Arch Gynecol Obstet 299: 525-532, 2019.

19. Cheng X, Feng Y, Wang X Wan X, Xie X and Lu W: The effectiveness of conization treatment for post-menopausal women with high-grade cervical intraepithelial neoplasia. Exp Ther Med 5: 185-188, 2013.

20. Messiou C, Morgan VA, De Silva SS, Ind TE and deSouza NM: Diffusion weighted imaging of the uterus: Regional ADC variation with oral contraceptive usage and comparison with cervical cancer. Acta Radiol 50: 696-701, 2009.

21. Mahon MM, Cox IJ, Dina R, Soutter WP, McIndoe GA, Williams AD and deSouza NM: (1)H magnetic resonance spectroscopy of preinvasive and invasive cervical cancer: In vivo-ex vivo profiles and effect of tumor load. J Magn Reson Imaging 19: 356-364, 2004.

22. Charles-Edwards EM, Messiou C, Morgan VA, De Silva SS, McWhinney NA, Katesmark M, Attygalle AD and DeSouza NM: Diffusion-weighted imaging in cervical cancer with an endovaginal technique: Potential value for improving tumor detection in stage Ia and Ib1 disease. Radiology 249: 541-550, 2008.

23. Patel-Lippmann K, Robbins JB, Barroilhet L, Anderson B, Sadowski EA and Boyum J: MR imaging of cervical cancer. Magn Reson Imaging Clin N Am 25: 635-649, 2017.

24. Stoehr A, Nann D, Staebler A, Oberlechner E, Brucker SY and Bachmann C: Difficulties in diagnosis of a minimal deviation adenocarcinoma of uterine cervix diagnosed postoperatively: Brief communication and literature review. Arch Gynecol Obstet 300: 1029-1043, 2019.

This work is licensed under a Creative Commons Attribution-NonCommercial-NoDerivatives 4.0 International (CC BY-NC-ND 4.0) License. 